\title{
VARIACIÓN ESTACIONAL DE HONGOS MICORRÍZICOS ARBUSCULARES ASOCIADOS CON Agave angustifolia Haw. EN LA SIERRA SONORENSE, MÉXICO
}

\section{SEASONAL VARIATION OF ARBUSCULAR MYCORRHIZAL FUNGI ASSOCIATED WITH Agave angustifolia Haw. AT THE SONORAN SIERRA, MÉXICO}

\author{
Andrés Ochoa-Meza ${ }^{1,2}$, Martín Esqueda $^{1^{*}}$, Rigel Fernández-Valle ${ }^{3}$ y Ricardo Herrera-Peraza ${ }^{3 \dagger}$
}

\begin{abstract}
${ }^{1}$ Coordinación de Tecnología de Alimentos de Origen Vegetal, Centro de Investigación en Alimentación y Desarrollo, A. C. Apdo. Postal 1735. 83000, Hermosillo, Sonora, México. Tel.: 01 (662) 289-2400 ext. 326; Fax: 01 (662) 280-0422. ${ }^{2}$ Departamento de Agricultura y Ganadería, Universidad de Sonora,. Apdo. Postal 54. 83000, Hermosillo, Sonora, México. ${ }^{3}$ Instituto de Ecología y Sistemática, Ministerio de Ciencia Tecnología y Medio Ambiente. Apdo. Postal 8029. 10800, La Habana, Cuba.
\end{abstract}

* Autor para correspondencia (esqueda@ciad.mx)

\section{RESUMEN}

Agave angustifolia Haw. se distribuye en la vertiente occidental de la Sierra Madre en Sonora, México, donde se utiliza para la elaboración del destilado denominado 'Bacanora'. Se muestreó rizosfera y raicillas de esta especie durante las cuatro estaciones del año, para evaluar su relación con hongos micorrízicos arbusculares (HMA) en tres poblaciones silvestres. La cantidad de esporas de HMA varió de 9200 a 43500 esporas $\mathrm{dm}^{-3}$, que pertenecen a 32 morfotipos de Acaulospora (10), Archaeospora (2), Diversispora (1), Glomus (17) y Pacispora (2). La colonización micorrízica fluctuó de 4.8 a $24.9 \%$, la densidad visual de 0.43 a $0.51 \%$, el micelio intrarradical de 3.8 a $8.9 \mathrm{mg} \mathrm{dm}^{-3}$, y el micelio extrarradical de 31.1 a $93.4 \mathrm{mg} \mathrm{dm}^{-3}$. El análisis de correspondencia mostró que sodio, potasio y magnesio son los factores abióticos más importantes, y que los factores bióticos relevantes fueron las relaciones micelio extrarradical:micelio intrarradical y micelio extrarradical:peso seco de raicillas. Sin embargo, el análisis de correspondencia canónica mostró que la relación $A$. angustifolia-HMA es más compleja y no puede explicarse solamente con estos factores.

Palabras clave: Agave angustifolia, Glomus, micorriza arbuscular, simbiosis.

\section{SUMMARY}

Agave angustifolia Haw. is distributed along the western slopes of the Sierra Madre in Sonora, México, where it is used for distilling a spirit called 'Bacanora'. Rhizosphere and rootlets of this species were sampled from three wild populations during the four seasons of the year, in order to evaluate their relationship with arbuscular mycorhizal fungi (AMF). The amount of AMF spores varied from 9200 to 43500 spores $\mathrm{dm}^{-3}$, which belong to 32 morphotypes of Acaulospora (10), Archaeospora (2), Diversispora (1), Glomus (17) and Pacispora (2). The mycorrhizal colonization ranged from 4.8 to 24.9 $\%$, the visual density from 0.43 to $0.51 \%$, the intraradical mycelium from 3.8 to $8.9 \mathrm{mg} \mathrm{dm}^{-3}$ and the extraradical mycelium from 31.1 to $93.4 \mathrm{mg} \mathrm{dm}^{-3}$. The correspondence analysis showed that sodium, potassium and magnesium were the most important abiotic factors, while the relevant biotic factors were the ratios of extraradical mycelium:intraradical mycelium and extraradical mycelium:rootlets dry weight. Nonetheless, the canonical correspondence analysis showed that $\boldsymbol{A}$. angustifolia-AMF interaction is far more complex and that it can not be explained solely by these factors.

Index words: Agave angustifolia, Glomus, arbuscular mycorrhiza, symbiosis.

\section{INTRODUCCIÓN}

De acuerdo con registros fósiles y datos moleculares, la asociación de plantas con hongos micorrízicos arbusculares (HMA) se remonta a más de 400 millones de años (Redecker, 2002). Algunos autores han sugerido que probablemente representa la asociación simbiótica más importante de la naturaleza y su coevolución con las plantas ha permitido que colonice alrededor de $80 \%$ de las especies vegetales (Brundrett, 2002; Brachmann y Parniske, 2006). Las ventajas de esta asociación se han documentado ampliamente en las últimas décadas (Gadkar et al., 2001; Jakobsen et al., 2002), donde destacan el aporte de los HMA a la mejora del estado hídrico y nutrición mineral de la planta, y ésta a cambio proporciona fotoasimilados a los hongos. El costo de carbono fijado para mantener una simbiosis verdadera generalmente varía entre etapas fenológicas y climáticas, de 4 a $20 \%$ (Van der Heijden y Sanders, 2002; Brachmann y Parniske, 2006).

La micotrofia, definida como la capacidad de las plantas para asociarse con HMA y obtener beneficios de esta relación, varía entre obligatoria, facultativa o ausente (Smith y Read, 1997). En el primer caso las plantas tienen un sistema radical magnolioide (raíces cortas y gruesas, con pocos pelos absorbentes o raicillas), mientras que las 
especies facultativas o no micotróficas presentan el sistema graminoide (gran cantidad de pelos absorbentes). En una micotrofia obligatoria el crecimiento de las plantas se reduce significativamente ante la ausencia de HMA. De acuerdo con Fitter (2004), la frecuencia y la longitud de pelos absorbentes en una raíz representan la mejor forma para evaluar la capacidad micotrófica de la planta. La presencia de pelos radicales indica la facultad de la planta para prosperar sin depender de la micorrización, y ambas características se correlacionan negativamente. No obstante, los resultados de varios estudios no son concluyentes e incluso algunas veces son contradictorios (Brundrett, 2002; Fitter, 2004).

En los ambientes áridos la asociación micorrízica parece crucial para la supervivencia de las plantas (Beena et al., 2000; Stutz et al., 2000), y por ello se han efectuado algunas investigaciones sobre la distribución de HMA en el Desierto de Sonora (Stutz y Morton, 1996; Stutz et al., 2000; Bashan et al., 2007), y sólo uno sobre HMA asociados con A. angustifolia (Armenta et al., 2003) a pesar de que este agave se distribuye en toda la Sierra de Sonora y sus poblaciones silvestres son materia prima para elaborar el mezcal 'Bacanora'. Armenta et al. (2003) encontró ocho morfotipos de esporas de HMA correspondientes a Acaulospora (1), Gigaspora (1) y Glomus (6), con presencia de colonización micorrízica a través de enrollamientos hifales, vesículas y arbúsculos, que son estructuras indicadoras de una simbiosis funcional; la mayor diversidad de HMA se encontró en un sitio ubicado en el límite de la distribución de $A$. angustifolia.

El objetivo del presente estudio fue analizar la variación estacional de los HMA asociados con $A$. angustifolia silvestre, así como los factores ambientales más importantes en dicha variación, en poblaciones ubicadas cerca del límite norte de distribución de la especie en la Sierra de Sonora, México.

\section{MATERIALES Y MÉTODOS}

\section{Localidades estudiadas}

La investigación se hizo en tres poblaciones silvestres de A. angustifolia: El Bajío, Localidad 1 (L1), (2947’28” LN, 109 00'28" LO; 912 msnm); Los Mochomos, Localidad 2 (L2), $\left(29^{\circ} 55^{\prime} 58^{\prime \prime}\right.$ LN, $109^{\circ} 00^{\prime} 04^{\prime \prime}$ LO; 925 msnm) y El Chorro, Localidad 3 (L3), (29 $42^{\prime} 45^{\prime}$ " LN, $109^{\circ} 08^{\prime} 21^{\prime \prime} \mathrm{LO}$; $892 \mathrm{msnm}$ ), todas ellas ubicadas en el municipio de Nácori Chico, Sonora, México. Estas localidades presentan un clima semiseco semicálido, con temperaturas extremas de -2 y $38^{\circ} \mathrm{C}$, con promedio de 18 a $22{ }^{\circ} \mathrm{C}$, y un régimen de lluvias de verano con precipitación entre 500 y $600 \mathrm{~mm}$ anuales. La topografía es compleja, con lomeríos medianos y altos, con pendientes de 2 a 17 grados (INEGI, 1981a). Los agaves se observaron asociados con árboles y arbustos, especialmente con Acacia cochliacantha Humb. \& Bonpl. ex Willd., Fouquieria mcdougalli Nash., Opuntia spp., Prosopis spp. y Sapium biloculare (S. Wats.) Pax, en vegetación de matorral subtropical asociado con matorral sarcocrasicaule (INEGI, 1981b).

\section{Muestreo}

En cada localidad se marcaron al azar tres cuadrantes de 50 x $100 \mathrm{~m}$; en cada cuadrante se estimó la población de agave y se muestreó al menos $10 \%$ de las plantas adultas, lo que dio en total 12,14 y 17 plantas para las localidades 1,2 y 3 respectivamente. Los criterios de inclusión de los agaves fueron una distancia mínima de separación de $0.75 \mathrm{~m}$ del tronco de otras especies vegetales y un tamaño mayor a $1 \mathrm{~m}$ de altura. Se excluyeron los agaves en floración o con el escapo floral cortado. Se obtuvo una muestra compuesta en cada cuadrante y tres mezclas de suelo por localidad.

De acuerdo con el criterio de Lutgen et al. (2003), quienes consideran que existe poca variación en los valores de micorrización durante la misma estación del año, la rizosfera de cada planta se dividió en cuatro secciones orientadas hacia los puntos cardinales. Se muestrearon las secciones a la mitad de la estación, iniciando en invierno con la sección sur y continuando en el sentido de las manecillas del reloj, para terminar en otoño con la sección este. La muestra se tomó desde $0.1 \mathrm{~m}$ del tronco hasta la mitad del radio de cobertura de la planta; se eliminó la capa superior y se colectó suelo y raicillas de una profundidad de 0.05 a $0.2 \mathrm{~m}$. La cantidad mínima de suelo obtenida por agave fue $750 \mathrm{~g}$, pues varió según la fase pedregosa. El suelo se repuso para evitar daños a las raíces expuestas. Las muestras se colocaron en bolsas de polietileno virgen de baja densidad y fueron transportadas en hieleras al laboratorio donde se secaron a temperatura ambiente, y su procesamiento se hizo a la brevedad posible.

\section{Análisis fisicoquímico de suelo}

En cada muestra de suelo se analizó: $\mathrm{pH}$, materia orgánica, conductividad eléctrica, textura, relación de adsorción de sodio (RAS), porcentaje de sodio intercambiable (PSI) y contenido y disponibilidad de nutrimentos, de acuerdo con las metodologías propuestas por Huerta-Rosas (1985). 


\section{Variables de micorrización}

Los análisis se hicieron en una alícuota de $100 \mathrm{~cm}^{3}$ de suelo secado al ambiente y tamizado con malla de $2 \mathrm{~mm}$. Las variables de micorrización se determinaron por duplicado en cada muestra compuesta. Se analizaron: cantidad de esporas de HMA, colonización micorrízica $(\mathrm{CM})$, densidad visual (DV), micelio intrarradical (MI), micelio extrarradical (ME), presencia de pelos radicales (PR), peso seco de raicillas (PSR) y las relaciones ME:MI y ME:PSR.

La desfloculación de las arcillas en los agregados del suelo se hizo mediante un tratamiento químico con $\mathrm{H}_{2} \mathrm{O}_{2}$ $1.5 \%$ por $1 \mathrm{~h}$, seguido de tamizado en húmedo y decantación a través de tamices de 125 y $44 \mu \mathrm{m}$; luego se lavó con agua corriente hasta eliminar la fracción menor de $44 \mu \mathrm{m}$ y se continuó con una desagregación física (centrifugación) a $1650 x g$ para la fracción $>125 \mu \mathrm{m}$ (fracción A) y a $3170 x g$ para la fracción $>44 \mu \mathrm{m}$ y $<$ $125 \mu \mathrm{m}$ (fracción B). Se decantó antes de pasar de nuevo a través del tamiz respectivo para eliminar las fracciones más densas, que corresponden a arena principalmente $\mathrm{y}$ que no contienen esporas ni micelio de HMA (Gerdemann y Nicolson, 1963; Herrera-Peraza et al., 2004). Las fracciones conservadas en los tamices se filtraron en papel Whatman \# 4 para eliminar el exceso de agua y se dejaron secar al ambiente por $24 \mathrm{~h}$ o hasta peso constante. En cada fracción se determinó peso seco y se separó una alícuota de $10 \%$ para la fracción A y de $5 \%$ para la fracción B, de donde se obtuvieron el número de esporas y su distribución por morfotipos.

Cuantificación de esporas de HMA. Para la extracción de esporas se centrifugó la alícuota $(5 \mathrm{~min}$ a
$1050 x g$ ) en un gradiente de agua:sacarosa $2 \mathrm{M}$, donde la interfase fue extraída y lavada para eliminar el exceso de sacarosa, y luego se midió la cantidad de esporas en microscopio estereoscópico (7 a $60 \mathrm{X})$, con el apoyo de una placa Doncaster. De cada morfotipo de esporas de HMA encontrado se hicieron preparaciones permanentes, montadas con polivinilglicol (PVLG) y reactivo de Melzer en PVLG 1:1. Posteriormente se identificaron y clasificaron hasta nivel de género, de acuerdo con Schenck y Pérez (1990), Morton et al. (1993) y el sitio http://invam.caf.wvu.edu. Los especímenes se registraron en la colección del Herbario Nacional del Instituto de Ecología y Sistemática IES-CITMA, La Habana, Cuba (HAC).

Determinación de colonización micorrízica (CM) y densidad visual (DV). Se procedió a extraer las raíces más finas mediante pinzas de disección, y se obtuvo $c a$. $200 \mathrm{mg}$ de muestra. Cada una se sometió a clarificación y tinción según Phillips y Hayman (1970). Una vez teñidas y con un peso conocido, se observaron al microscopio estereoscópico en una placa con retículo de $1.25 \mathrm{~cm}^{2}$, y se contaron las intersecciones de raicillas en las líneas necesarias ( $>100$ por medición), conforme al método de Giovannetti y Mosse (1980). El porcentaje de CM se calculó con la proporción de intersecciones micorrizadas con respecto al total de intersecciones registradas. Independientemente de la $\mathrm{CM}$, en las intersecciones se clasificó la DV en seis categorías de 0 a 5 (Figura 1). El número de intersecciones para cada categoría se multiplicó por $0,1.0,2.5,15.5,35.5$ y $47.5 \%$, y la DV fue la suma de todos los productos dividida entre el número total de intersecciones (Herrera-Peraza et al., 2004).

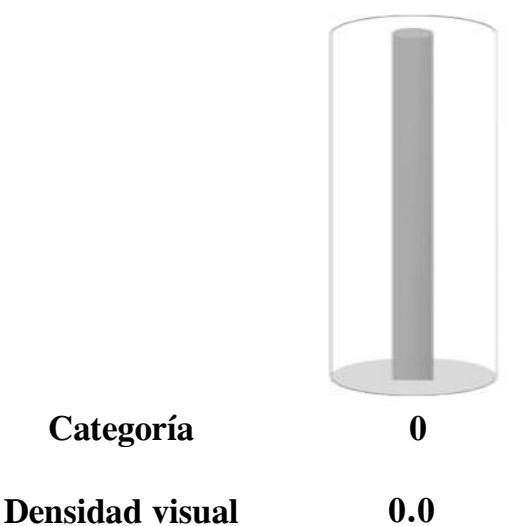

(\%)

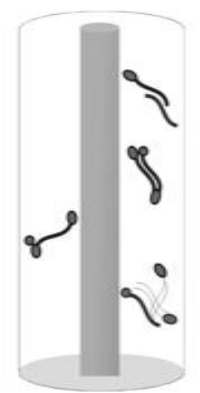

1

1.0

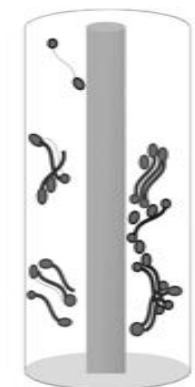

2

2.5

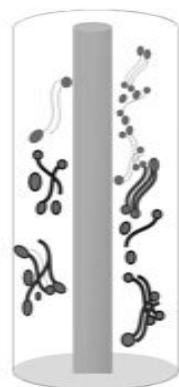

3

15.5

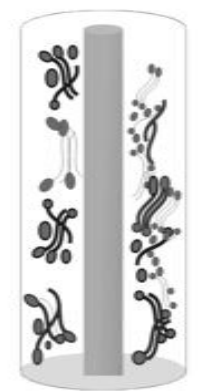

4

35.5

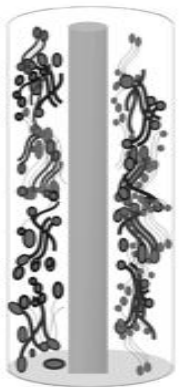

5

47.5

Figura 1. Categorías de densidad visual y porcentajes de ocupación fúngica estimados según Herrera-Peraza et al. (2004). 
Estimación de micelio intrarradical (MI) y micelio extrarradical (ME). El MI es igual a DV [PSR/100], expresado en $\mathrm{mg} \mathrm{dm}^{-3}$, con base en $20 \%$ de masa estimado para la corteza de una raicilla colonizada. El cilindro central se excluyó debido a que el HMA no lo coloniza y comúnmente constituye $80 \%$ del peso seco radical total. Para la determinación del ME se separaron alícuotas por duplicado entre 0.03 y $0.06 \mathrm{~g}$, y se dispersó la muestra de manera uniforme en una gota de glicerina sobre un portaobjetos. La preparación se revisó al microscopio compuesto a 100 ó $200 \mathrm{X}$ y se contabilizaron las hifas en cuatro líneas imaginarias (dos verticales y dos horizontales, en longitud y profundidad). El valor promedio obtenido para los cuatro conteos de líneas se multiplicó por la constante 0.000745 (Herrera-Peraza et al., 2004), lo que permitió estimar el peso (mg) del micelio extrarradical contenido en dicha alícuota. Dicha constante se estableció al multiplicar el total de líneas en el cubreobjetos de $22 \times 22 \mathrm{~mm}^{2}$ por el valor de una intercepción (1.57) y dividir el producto entre la longitud de la hifa en $1 \mathrm{mg}$ de micelio extraradical (42 146.26 $\mathrm{mm})$. La extrapolación del peso promedio de micelio extrarradical proveniente de dos alícuotas para cada fracción A y B permitió obtener los valores totales de MEA y ME-B, y la suma de éstos constituyó el estimado real de micelio extrarradical expresado en $\mathrm{mg} \mathrm{dm}^{-3}$.

Pelos radicales (PR), peso seco de raicillas (PSR), micelio extrarradical: micelio intrarradical (ME:MI) y micelio extrarradical: peso seco de raicillas (ME:PSR). La presencia o ausencia de pelos radicales se determinó en los segmentos de raicillas que interceptaban cada línea del retículo en las placas de conteo. Sus valores se expresaron en porcentaje con respecto al total de intersecciones contadas. El PSR se estableció al pesar las raicillas encontradas en una alícuota de $100 \mathrm{~cm}^{3}$ de suelo, las cuales se secaron a $60{ }^{\circ} \mathrm{C}$ hasta peso constante. Las relaciones ME:MI y ME:PSR se calcularon al dividir el valor de ME entre MI y PSR, respectivamente.

\section{Análisis estadístico}

Se hizo un análisis de varianza para cada variable con el paquete estadístico SPSS ver. 11. Las variables porcentuales se transformaron como sigue: a) Colonización micorrízica CM (\%), con Arcoseno (x); b) Micelio extrarradical, con $\log (\mathrm{x})$; y c) Número de esporas, con Log (x), como recomiendan Herrera-Peraza et al. (2004) para cumplir la condición de normalidad en la distribución. El análisis multivariado de los datos se hizo mediante el programa NTSYS-PC ver. 2.10j (Rohlf, 1993), al igual que el análisis de correspondencia (AC) entre factores bióticos y abióticos. Se utilizó el programa PC-ORD ver
4.41 (McCune y Mefford, 1999) para el análisis de correspondencia canónica (ACC) cuando el AC mostró fortaleza en las relaciones, con la finalidad de identificar la dependencia $o$ interdependencia entre los factores involucrados (McCune y Grace, 2004).

\section{RESULTADOS Y DISCUSIÓN}

Este es el primer estudio que se reporta sobre dinámica estacional de HMA asociados con $A$. angustifolia. Investigaciones previas han mostrado resultados sobre el efecto puntual de HMA en la asimilación de agua, nutrimentos y $\mathrm{CO}_{2}$ en Agave deserti Engelm. (Cui y Nobel, 1992), análisis de la composición de HMA y filamentosos asociados con A. angustifolia (Armenta et al., 2003), relación entre HMA y nodrizaje (Carrillo-García et al., 1999) y efecto de HMA sobre el enraizamiento de vitroplantas de Agave salmiana Otto (Rodríguez et al., 2007).

La densidad de población de $A$. angustifolia fue de 503, 202 y 199 individuos ha ${ }^{-1}$ en los sitios L3, L2 y L1 respectivamente. El grado de perturbación debido a las actividades antropogénicas fue disímil entre las localidades. En L3 la actividad de pastoreo de ganado vacuno es mínima; en L2 se observó la mayor perturbación debido a pastoreo de ganado, roturación del suelo y desmonte selectivo para el establecimiento de pastizales; y en L1 la actividad del pastoreo vacuno es mayor que en L3. La mayor densidad y desarrollo de individuos en L3 puede atribuirse al microclima existente en esa zona, debido a una protección natural dada por un lomerío bajo que repercute en mayor cobertura de vegetación. En las localidades muestreadas no existen estudios previos a la roturación del suelo y pastoreo, los cuales se han reportado como perturbaciones que afectan a los HMA (Smith y Read, 1997; Lovera y Cuenca, 2007). No obstante, al nivel del presente experimento en Sonora no se detectaron influencias atribuibles a estos factores sobre las variables estudiadas de los HMA.

\section{Análisis fisicoquímico del suelo}

Los suelos mostraron un $\mathrm{pH}$ ligeramente alcalino de 7.3 a 7.6 , y sin problemas de salinidad ni sodicidad, ya que la conductividad eléctrica fue $<2 \mathrm{dS} \mathrm{m}^{-1}$; la relación de adsorción de sodio fue menor a 1.7 y su porcentaje de sodio intercambiable inferior a 1.2 (Cuadro 1). El contenido de materia orgánica total fue de mediano a alto, varió de 3.2 en primavera a 4.1 en verano. El N-NO disponible fue similar en las estaciones del año, excepto en invierno donde aumentó hasta $2 \mathrm{mg} \mathrm{kg}^{-1}(\mathrm{P}<0.05)$, mientras que el contenido de $\mathrm{P}_{-} \mathrm{PO}_{4}^{-3}$ no cambió 
significativamente a lo largo del año. Las concentraciones de $\mathrm{K}, \mathrm{Ca}, \mathrm{Mg}, \mathrm{Cu}$ y $\mathrm{Mn}$ fluctuaron significativamente ( $\mathrm{P}$ $\leq 0.05$ ) durante las estaciones del año, contrario a lo que se observó en $\mathrm{Fe}, \mathrm{Zn}$ y $\mathrm{Na}$ en los que no se registró variación $(\mathrm{P}>0.05)$. La textura de las localidades fue migajón-areno-arcilloso (L1 y L2) y migajón-arenoso (L3) (datos no mostrados).

La fertilidad del suelo es baja, comparable a suelos de zonas áridas, donde aún cuando existen nutrimentos suficientes éstos se encuentran en forma insoluble (Castellanos et al., 2000). Las especies de Agave se distribuyen principalmente en suelos bien drenados con textura arenosa, sin problemas de salinidad o sodicidad (Nobel, 1988) como los observados en este estudio; no se detectaron síntomas de deficiencias nutricionales. Un contenido de mediano a alto de materia orgánica total refleja la productividad del ecosistema y sobre todo la lentitud del proceso de descomposición, el cual es únicamente favorecido por las precipitaciones de verano. Sin embargo, contrasta con los valores menores a $0.5 \%$ reportados generalmente para ambientes áridos (Castellanos et al., 2000).
Con base en el análisis de correspondencia se determinó que $\mathrm{Na}, \mathrm{K}, \mathrm{Mg}, \mathrm{P}_{-} \mathrm{PO}_{4}^{-3}$ y $\mathrm{Ca}$, influyeron significativamente en la asociación micorrízica. La aportación individual en la variación total de los dos primeros ejes cambió según la estación (Cuadro 2) y fue consistente con el orden de importancia. Na y $\mathrm{K}$ presentaron la mayor aportación absoluta en la variación; el primero con $62.5 \%$ en invierno y $47.0 \%$ en otoño; y el $\mathrm{K}$ tuvo $48.5 \%$ en primavera (Cuadro 2; Figura 2). La composición mineral es relevante en la asociación micorrízica y más aún con sequía, ya que impacta tanto las relaciones hídricas de la planta como la capacidad de retención de agua en el suelo (Auge et al., 2001). Esta simbiosis contribuye al mantenimiento de la turgencia celular y de la conductancia estomática cuando se presenta un déficit de agua, e incrementa la asimilación relativa del agua, la tasa de intercambio de $\mathrm{CO}_{2}$ y la eficiencia en el uso de agua, y propicia el ajuste osmótico celular (RuizLozano et al., 1995). Aunque no se determinó la contribución de la micorrización en la absorción de cada elemento, generalmente se acepta que los HMA favorecen la asimilación de elementos insolubles, v.g., fósforo y zinc (Johnson et al., 1997; Bucher, 2007).

Cuadro 1. Análisis fisicoquímico del suelo de invierno 2005 a otoño 2006.

\begin{tabular}{|c|c|c|c|c|}
\hline \multirow{2}{*}{ Factor edáfico } & \multicolumn{4}{|c|}{ Estación } \\
\hline & Invierno & Primavera & Verano & Otoño \\
\hline $\mathrm{pH}$ & $7.6 \pm 0.1 \mathrm{a}$ & $7.3 \pm 0.3 \mathrm{a}$ & $7.4 \pm 0.1 \mathrm{a}$ & $7.4 \pm 0.1 \mathrm{a}$ \\
\hline $\left.\mathrm{CE}(\mathrm{dS} \mathrm{m})^{-1}\right)^{(+)}$ & $1.6 \pm 0.15 b$ & $0.9 \pm 0.08 \mathrm{a}$ & $1.2 \pm 0.11 \mathrm{a}$ & $1.2 \pm 0.07 \mathrm{a}$ \\
\hline RAS $^{(+)}$ & $0.4 \pm 0.16 \mathrm{a}$ & $0.8 \pm 0.33 a$ & $1.5 \pm 0.15 b$ & $0.5 \pm 0.13 \mathrm{a}$ \\
\hline $\mathrm{PSI}^{(+1)}$ & $0.2 \pm 0.2 \mathrm{a}$ & $0.0 \pm 0.0 \mathrm{a}$ & $1.0 \pm 0.2 \mathrm{~b}$ & $0.1 \pm 0.1 \mathrm{a}$ \\
\hline Materia orgánica total (\%) & $3.9 \pm 0.5 \mathrm{a}$ & $3.2 \pm 0.39 \mathrm{a}$ & $4.1 \pm 0.48 \mathrm{a}$ & $3.9 \pm 0.25 \mathrm{a}$ \\
\hline $\mathrm{N}^{-\mathrm{NO}_{3}}{ }^{-}\left(\mathrm{mg} \mathrm{kg}^{-1}\right)$ & $2.0 \pm 0.51 \mathrm{~b}$ & $0.5 \pm 0.04 \mathrm{a}$ & $0.6 \pm 0.06 \mathrm{a}$ & $0.8 \pm 0.05 \mathrm{a}$ \\
\hline $\mathrm{P}-\mathrm{PO}_{4}^{-3}\left(\mathrm{mg} \mathrm{kg}^{-1}\right)$ & $85.6 \pm 40.5 \mathrm{a}$ & $63.9 \pm 23.1 \mathrm{a}$ & $62.2 \pm 21.8 \mathrm{a}$ & $41.5 \pm 10.4 \mathrm{a}$ \\
\hline $\mathrm{K}^{+}\left(\mathrm{cmol} \mathrm{kg}^{-1}\right)$ & $0.6 \pm 0.09 \mathrm{ab}$ & $0.5 \pm 0.06 \mathrm{ab}$ & $0.4 \pm 0.06 \mathrm{a}$ & $0.7 \pm 0.05 b$ \\
\hline $\mathrm{Ca}^{2+}\left(\mathrm{cmol} \mathrm{kg}^{-1}\right)$ & $12.1 \pm 1.5 \mathrm{~b}$ & $5.7 \pm 0.6 \mathrm{a}$ & $5.7 \pm 0.7 \mathrm{a}$ & $8.0 \pm 0.7 \mathrm{a}$ \\
\hline $\mathrm{Mg}^{2+}\left(\mathrm{cmol} \mathrm{kg}^{-1}\right)$ & $2.5 \pm 0.3 \mathrm{~b}$ & $1.4 \pm 0.2 \mathrm{a}$ & $2.6 \pm 0.2 \mathrm{~b}$ & $2.1 \pm 0.1 \mathrm{~b}$ \\
\hline $\mathrm{Fe}\left(\mathrm{mg} \mathrm{kg}^{-1}\right)$ & $12.4 \pm 1.3 \mathrm{a}$ & $14.5 \pm 2.6 \mathrm{a}$ & $13.7 \pm 1.5 \mathrm{a}$ & $14.8 \pm 1.4 \mathrm{a}$ \\
\hline $\mathrm{Cu}\left(\mathrm{mg} \mathrm{kg}^{-1}\right)$ & $2.1 \pm 0.1 \mathrm{a}$ & $1.9 \pm 0.1 \mathrm{a}$ & $2.7 \pm 0.2 \mathrm{~b}$ & $1.9 \pm 0.1 \mathrm{a}$ \\
\hline $\mathrm{Zn}\left(\mathrm{mg} \mathrm{kg}^{-1}\right)$ & $2.6 \pm 0.4 \mathrm{a}$ & $1.9 \pm 0.3 \mathrm{a}$ & $2.5 \pm 0.3 \mathrm{a}$ & $2.3 \pm 0.2 \mathrm{a}$ \\
\hline $\operatorname{Mn}\left(\mathrm{mg} \mathrm{kg}^{-1}\right)$ & $6.9 \pm 0.8 \mathrm{a}$ & $7.9 \pm 1.2 \mathrm{ab}$ & $10.9 \pm 1.4 \mathrm{c}$ & $7.9 \pm 0.6 \mathrm{abc}$ \\
\hline $\mathrm{Na}\left(\mathrm{mg} \mathrm{L}^{-1}\right)$ & $86.4 \pm 27.0 \mathrm{a}$ & $99.2 \pm 43.2 \mathrm{a}$ & $168.7 \pm 23.3 \mathrm{a}$ & $69.0 \pm 19.5 \mathrm{a}$ \\
\hline
\end{tabular}

${ }^{(†)} \mathrm{CE}=$ Conductividad eléctrica; ${ }^{(\#)}$ Relación de adsorción de sodio; ${ }^{(\#)}$ PSI = Porcentaje de sodio intercambiable. Los valores son promedio de 9 muestras \pm error estándar. Medias con letras iguales en una misma fila, no son estadísticamente diferentes (Duncan, 0.05). 
Cuadro 2. Porcentaje de contribución de los principales factores a la variación total en los dos primeros ejes del análisis de correspondencia.

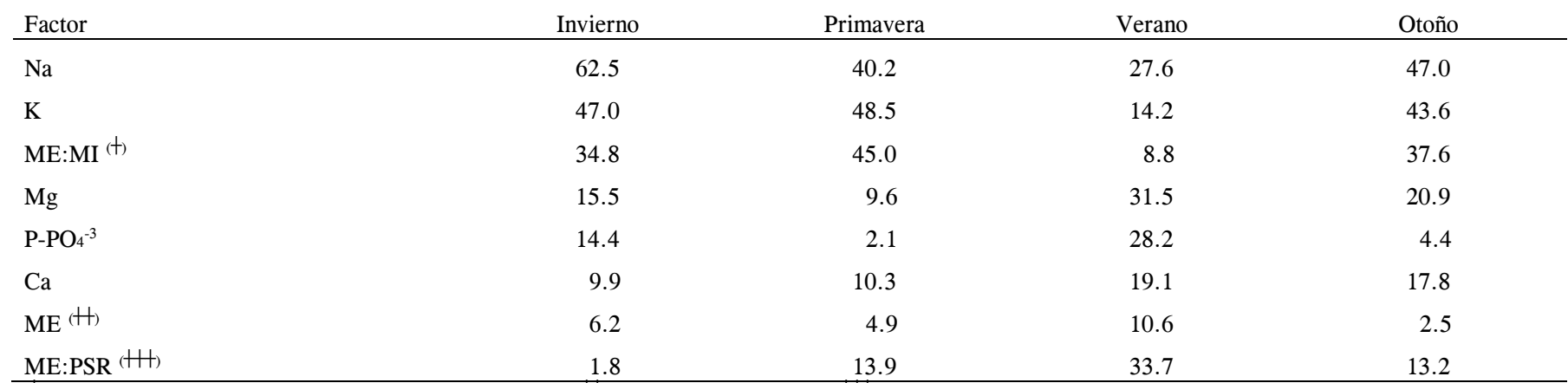

${ }^{(†)}$ Relación micelio extrarradical: micelio intrarradical; ${ }^{(\#)}$ Micelio extrarradical; ${ }^{(\#)}$ Relación micelio extrarradical: peso seco de raicillas.

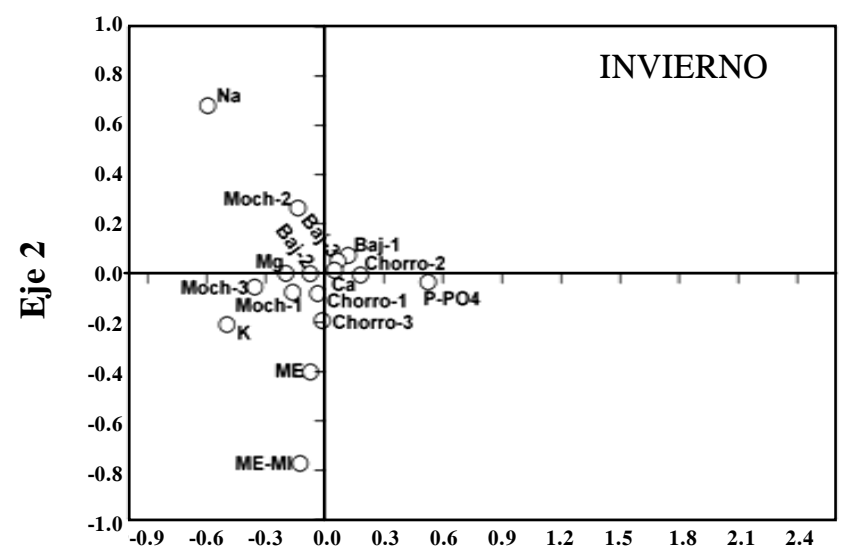

Eje 1

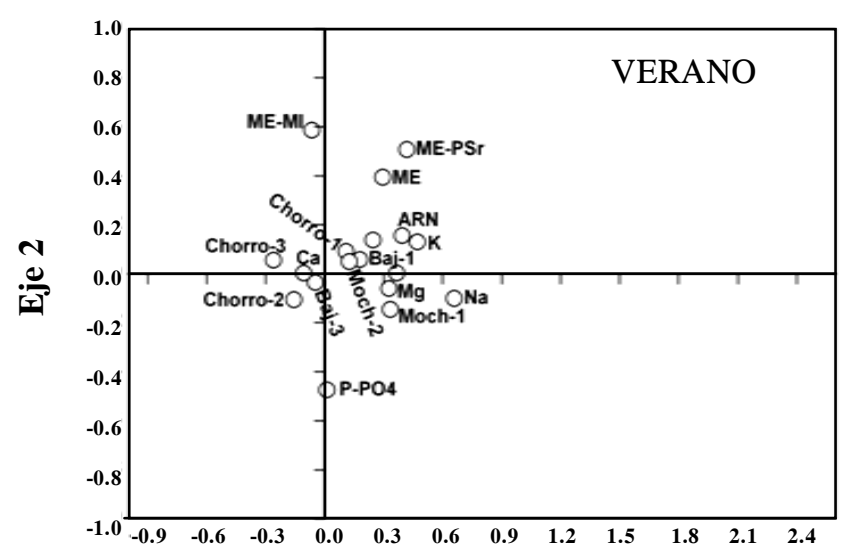

Eje 1

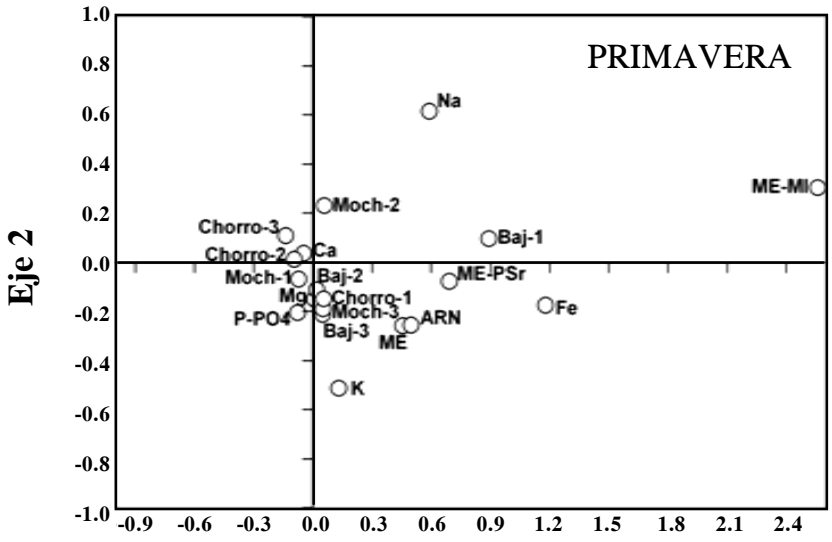

Eje 1

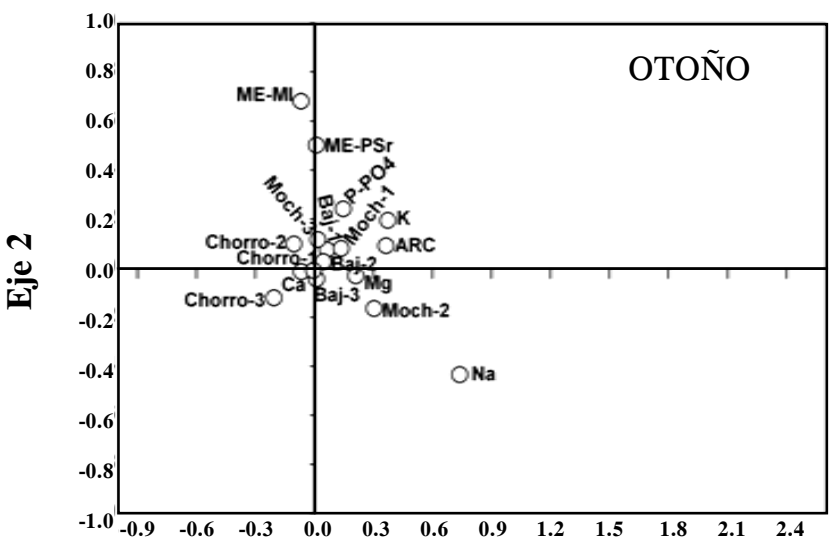

Eje 1

Figura 2. Contribución relativa y matriz de factores que intervienen estacionalmente en la relación Agave angustifolia-HMA. Na = Sodio; ME:MI = Relación micelio extrarradical: micelio intrarradical; ME:PSR = Micelio extrarradical: peso seco de raicillas; $\mathbf{A R N}=\mathbf{A r e n a}$; $\mathbf{A R C}=\mathbf{A r c i l l a}$; Moch 1, 2, 3 = Los Mochomos; Baj 1, 2, 3 = El Bajío; Chorro 1, 2, 3 = El Chorro. El número en la localidad indica el cuadrante.

\section{Variables de micorrización}

El número de esporas alcanzó su valor mínimo de 9200 a 9500 esporas $\mathrm{dm}^{-3}$ en invierno y primavera, y el máximo en verano con 43500 esporas $\mathrm{dm}^{-3}$. De acuerdo con Camargo-Ricalde et al. (2005), no hay relación directa entre la proporción de fotoasimilados aportados por la planta y el grado de esporulación del hongo, y este último 
puede ser afectado por factores diversos como la identidad del hospedero y el resto de la comunidad microbiana, entre otros. Para promover la esporulación se sugiere someter a estrés a las plantas trampa, especialmente en especies difíciles de esporular. Además, existe una relación entre la senescencia de la planta y la reproducción de los HMA a través de esporas (Morton et al., 1993).

En $A$. angustifolia no se probaron los argumentos anteriores. Sin embargo, la variación en el nivel de estrés hídrico fue evidente a lo largo del año. La mayor esporulación (verano) coincidió con temperaturas benignas para el desarrollo del agave (Nobel, 1988; PimientaBarrios et al., 2003) y la disponibilidad de agua provista por lluvias. Lo anterior no correlacionó con la disponibilidad de nutrientes, y la temperatura tuvo un efecto más determinante en la esporulación de HMA no aparentemente asociados con el agave. La cantidad de esporas es superior a la mayoría de los estudios existentes para zonas áridas y semiáridas. Bashan et al. (2007) reportaron de 200 a 700 esporas $\mathrm{dm}^{-3}$ en la asociación con Fouquieria columnaris (Kell.) Kell. ex Curran. Cui y Nobel (1992) encontraron de 0 a 8 esporas $\mathrm{dm}^{-3}$ con $A$. deserti; en ambientes húmedos, Guadarrama y ÁlvarezSánchez (1999) observaron de 38 a 238 esporas $\mathrm{dm}^{-3}$.

Los 32 morfotipos de esporas de HMA se clasificaron hasta género, con excepción de Glomus sinuosum (Gerd. \& B.K. Bakshi) R.T. Almeida \& N.C. Schenck, Acaulospora (10), Archaeospora (2), Diversispora (1), Glomus (16) y Pacispora (2) (Figura 3). La esporulación es un proceso particularmente diferente para cada HMA. La presencia o ausencia de esporas en una época del año está más relacionada con la fisiología del hongo (Pringle y Bever, 2002; Lutgen et al., 2003) e influenciada por la disponibilidad de fotoasimilados del hospedero (Morton et al., 1993). En este estudio no se tomaron en cuenta los conteos de esporas para evaluar la interacción plantaHMA, sino únicamente para su caracterización morfológica e identificación.

La presencia de 32 morfotipos contrasta con la baja diversidad de HMA observada en estudios previos en regiones áridas y semiáridas con plantas silvestres, v.g., de 3 a 12 morfotipos (Cui y Nobel, 1992; Stutz y Morton, 1996; Armenta et al., 2003) y en pocos casos de 15 a 25 (Guadarrama y Álvarez-Sánchez, 1999; Camargo-Ricalde y Esperón-Rodríguez, 2005; Bashan et al., 2007; Lovera y Cuenca, 2007). Así mismo, se contrapone a la idea generalmente aceptada de que la diversidad de HMA es baja en ambientes áridos (Carrillo-García et al., 1999). Un aspecto similar a los estudios anteriores se basa en la prevalencia de morfotipos correspondientes a Glomus, género que comprendió $53 \%$ del total de muestreos en Sonora.

En Fouquieria columnaris comprende $52 \%$ del total de morfotipos (Bashan et al., 2007), y un porcentaje similar se registró en Mimosa lacerata Rose, M. luisana Brandegee y $M$. polyantha Benth., y con formación de conglomerados en ambientes semiáridos de Tehuacán, México (Camargo-Ricalde y Esperón-Rodríguez, 2005). Armenta et al. (2003) reportó ocho morfotipos correspondientes a Glomus (6), Acaulospora (1) y Gigaspora (1) asociados con $A$. angustifolia. Esta diversidad comparada con el estudio actual, podría deberse a que son localidades diferentes, al muestreo durante las cuatro estaciones y a la variación en el nivel de precipitación (datos no mostrados). En el presente estudio no se observaron morfotipos de Gigaspora y Scutellospora, este último registrado por Bashan et al. (2007) en ambientes más áridos. Contrariamente, en este último estudio no encontraron especies de Acaulospora, las cuales en Sonora ocupan $c a .33 \%$ del total de esporas aparentemente asociadas con $A$. angustifolia, y un porcentaje más alto que en Mimosa spp. en Tehuacán, México (Camargo-Ricalde y Esperón-Rodríguez, 2005).

Se reconoce la falta de especificidad de los HMA (Jakobsen et al., 2002; Klironomos, 2003), debido a que en más de $96 \%$ de los casos se ha logrado establecer la micorrización. Van der Heijden et al. (1998) demostraron las diferencias en especificidad entre plantas y HMA los cuales tuvieron un papel distinto en cada ecosistema. El estudio que aquí se reporta podría evidenciar que $A$. angustifolia requiere de la asociación con HMA y que existe una dinámica funcional en esta simbiosis a lo largo del año reflejada en la variación estacional de morfotipos y nivel de ocupación de la micorriza en la raíz.

La colonización micorrízica $(\mathrm{CM})$ varió de $4.8 \%$ en invierno hasta $24.9 \%$ en primavera (Cuadro 3), con valores similares en primavera, verano y otoño $(\mathrm{P}>$ 0.05). Con excepción de invierno, la CM mostró valores superiores a los registrados para $A$. deserti (1.5 a $10 \%)$ en el Desierto Sonorense por Cui y Nobel (1992). PimientaBarrios et al. (2003) determinaron una CM de 15 a $30 \%$ en Opuntia robusta Wendl., sin limitaciones de nutrimentos. En $A$. angustifolia los resultados difieren parcialmente de lo establecido sobre el aumento de la CM debido a estrés hídrico (Auge, 2001), ya que si bien la CM aumenta en la época más seca (primavera), se mantiene relativamente constante durante el resto del año. En Sonora la variable ambiental más influyente podría corresponder a la temperatura, que limitaría la emisión de raicillas a pesar de la presencia de lluvias en invierno. 

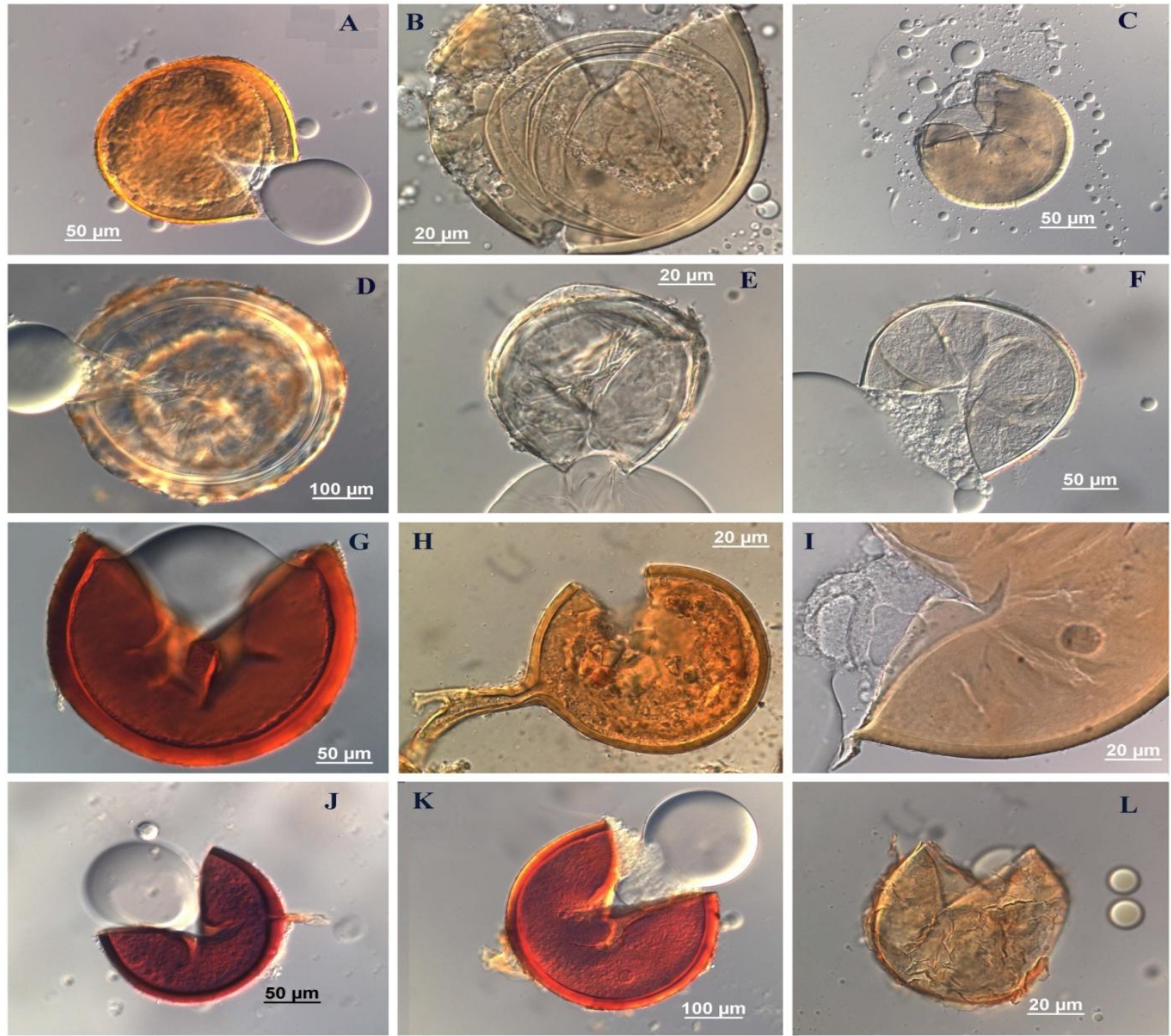

Figura 3. Morfotipos de HMA asociados con Agave angustifolia. A) Acaulospora sp. 1, B) Acaulospora aff. scrobiculata, C) Acaulospora aff. laevis, D) Archaeospora sp. 1, E) Archaeospora sp. 2, F) Diversispora sp. 1, G) Glomus sp. 1, H) Glomus aff. heterosporum, I) Glomus aff. claroideum, J) Glomus sp. 2, K) Glomus sp. 3, L) Pacispora sp. 1.

La densidad visual (DV) se mantuvo constante durante el periodo de muestreo, con valores desde $0.43 \%$ en otoño hasta $0.51 \%$ en primavera. Estos resultados podrían evidenciar un control de $A$. angustifolia sobre el crecimiento interno del HMA. Los valores bajos de DV no implican necesariamente que la relación simbiótica sea funcional en mayor o menor grado, ya que el intercambio neto entre los simbiontes no es proporcional a ésta $u$ otras variables como colonización micorrízica y micelio extrarradical (Herrera-Peraza et al., 2004).

El micelio intrarradical (MI) cambió de 3.8 hasta 8.9 $\mathrm{mg} \mathrm{dm}{ }^{-3}$ en otoño y primavera respectivamente, y tuvieron una correlación positiva con pelos radicales en otoño $(r=$ 0.97) y negativa en verano $(r=-0.98)$. La presencia de micelio extrarradical fue similar entre las estaciones, excepto en otoño donde se detectó un aumento $(\mathrm{P}<0.05)$ de $c a .2 .5$ veces (Cuadro 3). Este incremento posterior a la temporada de lluvias podría estar influenciado por la mayor disponibilidad de agua que favorece los procesos metabólicos de la planta, en conjunto con un régimen de temperatura apropiado (Nobel, 1988). Lutgen et al. (2003), al trabajar en un pastizal con una precipitación 50 a $55 \%$ menor que en Sonora, encontraron que la cantidad de micelio extrarradical disminuyó de 82 a $50 \mathrm{~m} \mathrm{~g}^{-1}$ de suelo al pasar de la época de lluvia a la de sequía. En $A$. angustifolia se observó un comportamiento similar, aunque la longitud del micelio fue menor de 0.4 a $2.2 \mathrm{~m} \mathrm{~g}^{-1}$ de suelo en verano y otoño respectivamente (datos no mostrados). 
Cuadro 3. Variables de micorrización en Agave angustifolia silvestre de invierno 2005 a otoño 2006.

\begin{tabular}{lcccc}
\hline & Invierno & Primavera & Verano & Otoño \\
\hline Esporas x $10^{3} \mathrm{dm}^{-3}$ & $9.20 \pm 3.20 \mathrm{a}$ & $9.50 \pm 2.10 \mathrm{a}$ & $43.50 \pm 8.40 \mathrm{c}$ & $23.90 \pm 3.80 \mathrm{~b}$ \\
Colonización micorrízica (\%) & $4.80 \pm 1.20 \mathrm{a}$ & $24.90 \pm 3.70 \mathrm{~b}$ & $21.90 \pm 2.10 \mathrm{~b}$ & $22.60 \pm 1.90 \mathrm{~b}$ \\
Densidad visual (\%) & $0.50 \pm 0.26 \mathrm{a}$ & $0.51 \pm 0.10 \mathrm{a}$ & $0.45 \pm 0.08 \mathrm{a}$ & $0.43 \pm 0.06 \mathrm{a}$ \\
Peso seco raicillas (PSR), $\mathrm{mg} \mathrm{dm}^{-3}$ & $7.74 \pm 1.05 \mathrm{c}$ & $0.60 \pm 0.07 \mathrm{a}$ & $0.56 \pm 0.07 \mathrm{a}$ & $3.75 \pm 0.68 \mathrm{~b}$ \\
& & & & \\
Pelos radicales (\%) & $0.09 \pm 0.02 \mathrm{a}$ & $0.08 \pm 0.02 \mathrm{a}$ & $0.17 \pm 0.03 \mathrm{~b}$ & $0.29 \pm 0.04 \mathrm{c}$ \\
Micelio intrarradical (MI), $\mathrm{mg} \mathrm{dm}^{-3}$ & $5.80 \pm 0.90 \mathrm{~b}$ & $8.90 \pm 2.70 \mathrm{~b}$ & $6.90 \pm 2.30 \mathrm{~b}$ & $3.80 \pm 1.20 \mathrm{a}$ \\
Micelio extrarradical (ME), $\mathrm{mg} \mathrm{dm}^{-3}$ & $33.90 \pm 5.20 \mathrm{a}$ & $37.80 \pm 4.30 \mathrm{a}$ & $31.10 \pm 5.10 \mathrm{a}$ & $93.40 \pm 9.30 \mathrm{~b}$ \\
Relación ME:MI, mg mg & $6.50 \pm 1.60 \mathrm{~b}$ & $4.20 \pm 0.10 \mathrm{a}$ & $5.10 \pm 1.60 \mathrm{~b}$ & $24.40 \pm 1.00 \mathrm{c}$ \\
Relación ME:PSR, $\mathrm{mg} \mathrm{mg}^{-1}$ & $5.00 \pm 1.10 \mathrm{a}$ & $72.20 \pm 11.7 \mathrm{c}$ & $58.50 \pm 9.00 \mathrm{c}$ & $33.90 \pm 8.10 \mathrm{~b}$ \\
\hline
\end{tabular}

Los valores son promedio de 9 muestras \pm error estándar. Medias con letras iguales en una misma fila, no son estadísticamente diferentes (Duncan, 0.05 ).

El peso seco de raicillas (PSR) fue menor en primavera y verano, y aumentó $(\mathrm{P}<0.05)$ en otoño e invierno (Cuadro 3). El valor más alto del PSR se observó en invierno, cuando se registró el menor porcentaje de colonización micorrízica. La emisión de nuevas raicillas aumenta rápidamente antes de la época de lluvias (Nobel, 1988). Aunque las raicillas presentan pelos radicales, éstos se reducen rápidamente cuando la raíz engrosa y se lignifica (Nobel, 1997). Así, el porcentaje más bajo de pelos radicales se registró en primavera e invierno, las estaciones más secas del año. Fitter et al. (1991) y Fitter (2004) notaron que la presencia de pelos absorbentes hace menos dependiente a la planta de la micorrización. Sin embargo, su presencia no indica que la planta carece de micotrofia. Fitter (2004) estableció que los pelos radicales e hifas de los HMA cumplen la misma función, aunque no existe una relación directa entre micelio extrarradical y pelos radicales. La edad de la raíz también podría influenciar la presencia de pesos radicales; una de las estrategias de agaváceas y cactáceas es la pérdida de raicillas a medida que aumenta el estrés hídrico (Nobel, 1988), mientras que el micelio extrarradical (ME) está determinado por la disponibilidad de nutrimentos en el suelo (Johnson et al., 1997; Lutgen et al., 2003).

La relación micelio extrarradical:micelio intrarradical (ME:MI) varió de 4.2 en primavera hasta 24.4 en otoño, y la razón micelio extrarradical:peso seco de raicillas (ME:PSR) varió de 5.0 en invierno hasta 72.2 en primavera (Cuadro 2). La relación ME:MI se ha reportado como un indicador del costo de la simbiosis. Ambos organismos buscan el equilibrio en la relación costo beneficio, la cual se presenta generalmente en sistemas poco o nada perturbados (Johnson et al., 1997). Los resultados obtenidos en $A$. angustifolia confirman lo anterior y reflejan la variación estacional de esta relación acorde con las condiciones ambientales, particularmente con la disponibilidad de agua.

El análisis de correspondencia mostró que las relaciones ME:MI y ME:PSR son las más influyentes de los factores bióticos, con efectos hasta de $45 \%$ y $33.7 \%$ en primavera y verano, respectivamente (Cuadro 3). Herrera-Peraza et al. (2004) establecieron que las relaciones ME:MI y ME:PSR definen una parte importante en la relación planta:HMA. Por ejemplo, valores altos de ME:MI indican una tendencia austera, la cual implica que una cantidad mayor de HMA explora el suelo, y una menor crece en estructuras intercelulares de la raíz. Aunque el análisis de correspondencia mostró relaciones entre la disponibilidad de elementos en el suelo y las variables de micorrización, en el análisis de correspondencia canónica no se detectaron las relaciones esperadas. Esto indica que la interacción $A$. angustifoliaHMA es compleja y no puede explicarse con base en uno o pocos factores, lo cual es congruente con estudios previos en otras especies (Van der Heijden y Sanders, 2002; Klironomos, 2003; Lutgen et al., 2003).

\section{CONCLUSIONES}

En la rizosfera de $A$. angustifolia se observó una cantidad y diversidad alta de morfotipos de HMA, lo cual contrastó con los estudios previos en ecosistemas áridos. Las variables de micorrización mostraron un cambio estacional, al aumentar la esporulación en el verano y el micelio extrarradical en el otoño, así como una disminución en el micelio intrarradical en el otoño, lo cual sugiere una diversidad funcional entre los HMA. El análisis de correspondencia mostró que la disponibilidad de elementos como sodio, potasio y magnesio son los factores abióticos más importantes en la micorrización, y en los 
bióticos fueron las relaciones micelio extrarradical:micelio intrarradical y el micelio intrarradical:peso seco de raicillas. No obstante, el análisis de correspondencia canónica indicó que la relación $A$. angustifolia-HMA es más compleja y no puede explicarse solamente con estos factores.

\section{AGRADECIMIENTOS}

Este trabajo está dedicado a la memoria del maestro, colega, amigo y coautor Dr. Ricardo Herrera-Peraza, micólogo destacado a nivel internacional. Los autores agradecen a Sergio Moreno, Alfonso Sánchez, Aldo Gutiérrez y Francisco Coronado por su asistencia en campo y logística, a Yamir Torres por su apoyo en el laboratorio y a Támine González por elaborar la Figura 1. Se agradece también el apoyo recibido a través de los proyectos FOMIX Gobierno del Estado de SonoraCONACYT (SON-2003-C01-5) e IAI-CRN II-14, US-NSF (GEO-04523250). AOM agradece el apoyo del CONACYT en sus estudios de posgrado.

\section{BIBLIOGRAFÍA}

Armenta A D, A Sánchez, T Cervantes, I Higuera, M Esqueda (2003) Hongos filamentosos y micorrízicos asociados con Agave angustifolia Haw. Boletín CIAD 12:1-2.

Auge R M (2001) Water relations, drought and vesicular-arbuscular mycorrhizal symbiosis. Mycorrhiza 11:3-42.

Bashan Y, T Khaosaad, B G Salazar, J A Ocampo, A Wiemken, F Oehl, H Vierheilig (2007) Mycorrhizal characterization of the boojum tree, Fouquieria columnaris, an endemic ancient tree from the Baja California Peninsula, Mexico. Trees 21:329-335.

Beena K R, N S Raviraja, A B Arun, K R Sridhar (2000) Diversity of arbuscular mycorrhizal fungi on the coastal sand dunes of the west coast of India. Curr. Sci. 79:1459-1466.

Brachmann A, M Parniske (2006) The most widespread symbiosis on earth. Plos Biology 4:1111-1112.

Brundrett M C (2002) Coevolution of roots and mycorrhizas of land plants. New Phytol. 154:275-304.

Bucher M (2007) Functional biology of plant phosphate uptake at root and mycorrhiza interfaces. New Phytol. 173:11-26.

Camargo-Ricalde S, M Esperón-Rodríguez (2005) Efecto de la heterogeneidad espacial y estacional del suelo sobre la abundancia de esporas de hongos micorrizógenos arbusculares en el valle semiárido de Tehuacán-Cuicatlán, México. Rev. Biol. Trop. 53:339-352.

Carrillo-García A, J L León-de la Luz, Y Bashan, G Bethlenfalvay (1999) Nurse plants, mycorrhizae, and plant establishment in a disturbed area of the Sonoran Desert. Restoration Ecol. 7:321335 .

Castellanos J, J X Uvalle-Bueno, A Aguilar-Santelises (2000) Manual de Interpretación de Análisis de Suelos y Aguas. INCAPA. Guanajuato, México. 226 p.

Cui M, P S Nobel (1992) Nutrient status, water uptake and gas exchange for three desert succulents infected with mycorrhizal fungi. New Phytol. 122:643-649.

Fitter A H (2004) Magnolioid roots-hairs, architecture and mycorrhizal dependency. New Phytol. 164:15-16.
Fitter A H, T R Stickland, M L Harvey, G W Wilson (1991) Architectural analysis of plant root systems 1. Architectural correlates of exploitation efficiency. New Phytol. 118:375-382.

Gadkar V, R David-Schwartz, T Kunik, Y Kapulnik (2001) Arbuscular mycorrhizal fungal colonization: Factors envolved in host recognition. Plant Physiol. 127:1493-1499.

Gerdemann J W, T H Nicolson (1963) Spores of mycorrhizal Endogone species extracted from soil by wet-sieving and decanting. Trans. Brit. Mycol. Soc. 46:235-244.

Giovannetti M, B Mosse (1980) An evaluation of techniques for measuring vesicular-arbuscular mycorrhizal infection in roots. New Phytol. 84:489-500.

Guadarrama, P, F J Álvarez-Sánchez (1999) Abundance of arbuscular mycorrhizal fungi spores in different environments in a tropical rain forest, Veracruz, Mexico. Mycorrhiza 8:267-270.

Herrera-Peraza, R A, E Furrazola, R L Ferrer, R Fernandez-Valle, $Y$ Torres-Arias (2004) Functional strategies of root hairs and arbuscular mycorrhizae in an evergreen tropical forest, Sierra del Rosario, Cuba. Rev. CENIC Cien. Biol. 35:113-123.

Huerta-Rosas R (1985) Propiedades Físicas y Químicas de Suelos Manual de Laboratorio. Universidad de Guadalajara. Guadalajara, México. 143 p.

INEGI (1981a) Guías para la Interpretación de Cartografía. Geología. INEGI. México, D.F. 32 p.

INEGI (1981b) Guías para la Interpretación de Cartografía. Uso del Suelo. INEGI. Aguascalientes, México. 49 p.

Jakobsen I, S E Smith, F A Smith (2002) Function and diversity of arbuscular mycorrhizae in carbon and mineral nutrition. In: Mycorrhizal Ecology. M G van der Heijden, I R Sanders (eds). Springer-Verlag. Berlin, Alemania. pp:75-92.

Johnson N C, J H Graham, F A Smith (1997) Functioning of mycorrhizal associations along the mutualism-parasitism continuum. New Phytol. 135:575-585.

Klironomos J N (2003) Variation in plant response to native and exotic arbuscular mycorrhizal fungi. Ecology 84:2292-2301.

Lovera M, G Cuenca (2007) Diversity of arbuscular mycorrhizal fungi (AMF) and mycorrhizal potential of the soil from a natural and a disturbed savannah from La Gran Sabana, Venezuela. Interciencia 32:108-114.

Lutgen E R, D Muir-Clairmont, J Graham, M C Rillig (2003) Plant seasonality of arbuscular mycorrhizal hyphae and glomalin in a western Montana grassland. Plant and Soil 257:71-83.

McCune B, J Grace (2004) Analysis of Ecological Communities. MJM Software. Gleneden Beach. Oregon, USA.

McCune B, M J Mefford (1999) PC-ORD, Multivariate Analysis of Ecological Data, Version 4.41. MjM software design. Gleneden Beach. Oregon, USA.

Morton J B, S P Bentivenga, W W Wheeler (1993) Germ plasm in the international collection of arbuscular and vesicular-arbuscular mycorrhizal fungi (INVAM) and procedures for culture development, documentation and storage. Mycotaxon 48:491528

Nobel P S (1988) Environmental Biology of Agaves and Cacti. Cambridge University Press. New York, USA. 270 p.

Nobel P S (1997) Root distribution and seasonal production in the northwestern Sonoran desert for a C3 subshrub, a C4 bunchagrass and a CAM leaf succulent. Amer. J. Bot. 84:949955

Phillips J M, D S Hayman (1970) Improved procedures for clearing roots and staining parasitic and vesicular-arbuscular mycorrhizal fungi for rapid assessment of infection. Trans. Brit. Mycol. Soc. 55:158-161.

Pimienta-Barrios E, M Del Castillo-Aranda, A Munoz-Urias, P S Nobel (2003) Effects of benomyl and drought on the mycorrhizal development and daily net $\mathrm{CO}_{2}$ uptake of a wild platyopuntia in a rocky semi-arid environment. Ann. Bot. 92:239-245. 
Pringle A, J D Bever (2002) Divergent phenologies may facilitate the coexistence of arbuscular mycorrhizal fungi in a North Carolina grassland. J. Bot. 89:1439-1446.

Redecker D (2002) Molecular identification and phylogeny of arbuscular mycorrhizal fungi. Plant and Soil 244:67-73.

Rodríguez G, F Morales, R Gutiérrez, S Aguilar, E Pérez (2007) Generación de raíces transformadas de Agave salmiana Otto y su colonización por Glomus intraradices. Rev. Fitotec. Mex. 30:215-222.

Rohlf F J (1993) NTSYS-pc, Numerical Taxonomy and Multivariate Analysis System, Version 2.10j. Applied Biostatistics Inc. Exeter Software. New York, USA.

Ruiz-Lozano J M, R Azcón, M Gómez (1995) Effects of arbuscular mycorrhizal Glomus species on drought tolerance:physiological and nutritional plant responses. Appl. Environ. Microbiol. 61:456-460.

Schenck N C, Y Pérez (1990) Manual for the Identification of VA Mycorrhizal Fungi. 3rd ed. Synergistic Publications. Gainesville, USA. $286 \mathrm{p}$.
Smith, S E, DJ Read (1997) Mycorrhizal Symbiosis. 2nd ed. Academic Press. San Diego, USA. 605 p.

Stutz J C, J B Morton (1996) Successive pot cultures reveal high species richness of arbuscular endomycorrhizal fungi in arid ecosystems. Can. J. Bot. 74:1883-1889.

Stutz J C, R Copeman, C A Martin, J B Morton (2000) Patterns of species composition and distribution of arbuscular mycorrhizal fungi in arid regions of southwestern North America and Namibia, Africa. Can. J. Bot. 78:237-245.

Van der Heijden M G A, I R Sanders (2002) Mycorrhizal Ecology. Springer. Berlin, Alemania. 469 p.

Van der Heijden M G A, T Boller, A Wiemken, I R Sanders (1998) Different arbuscular mycorrhizal fungal species are potential determinants of plant community structure. Ecology 79:20822091. 